\title{
First Case of Chromobacterium violaceum as Urinary Tract Infection Agent in Angola
}

\author{
Aleksey Shatalov ${ }^{1}, Z_{i v}$ Maianski $^{2}$ \\ ${ }^{1}$ Laboratory of Microbiology, Luanda Medical Center, Luanda, Angola \\ ${ }^{2}$ Urology Department, Luanda Medical Center, Luanda, Angola \\ Email: alshatalov@gmail.com
}

How to cite this paper: Shatalov, A. and Maianski, Z. (2019) First Case of Chromobacterium violaceum as Urinary Tract Infection Agent in Angola. Open Journal of Medical Microbiology, 9, 37-40. https://doi.org/10.4236/ojmm.2019.91005

Received: February 22, 2019

Accepted: March 22, 2019

Published: March 25, 2019

Copyright $\odot 2019$ by author(s) and Scientific Research Publishing Inc. This work is licensed under the Creative Commons Attribution International License (CC BY 4.0).

http://creativecommons.org/licenses/by/4.0/

\section{(c) (i) Open Access}

\begin{abstract}
Background: Chromobacterium violaceum is a Gram-negative, a facultative anaerobe bacteria producing violacein pigment. C. violaceum is generally present as the normal flora of water and soil. The Urine Tract Infection (UTI) due to $C$. violaceum is very rare. Until now there was no report from Africa about UTIs caused by $C$. violaceum. The antimicrobial susceptibility pattern of $C$. violaceum is very limited due to the rarity of isolation from clinical specimens. Here, we describe the first case of urinary tract infection caused by $C$. violaceum in Angola. Aim: Our case report was carried out to assess the sensitivity and resistance pattern of $C$. violaceum as the causative agent of UTI. Results: C. violaceum was sensitive to Amoxicillin/Clavulanic acid, Ceftriaxon, Ciprofloxacin, Doxycycline, Trimethoprim/Sulfamethoxazole, Piperacilin/Tazobactam, Gentamicin, Amikacin, Aztreonam and imipenem. The bacteria showed resistance to Cefuroxime. Conclusion: Here, we report a rare case of complicate urinary tract infection caused by $C$. violaceum in patient, who was treated successfully with ciprofloxacin for a total duration of 7 days.
\end{abstract}

\section{Keywords}

Chromobacterium violaceum, Lactose Non-Fermenting, Urine Tract Infection (UTI), Antibiotic Susceptibility

\section{Introduction}

Chromobacterium violaceum, is a Gram-negative, facultative anaerobe bacteria producing violacein pigment. C. violaceum is generally present as the normal flora in water and soil, and very rare causes human skin lesions, sepsis and urinary tract infections [1] [2] [3] [4]. C. violaceum infection is a rare emerging infection with a high mortal rate [5]. Nearly one hundred cases of $C$. violaceum have been reported around the world, mainly from tropical and subtropical 
areas of Asia, South America, Australia, and southeastern United States, including three cases from Africa [5]. Here, we report a case of $C$. violaceum in a patient (36 years old) suffering a complicated urine tract infection. This is the first case of $C$. violaceum as the UTI agent infection reported in Angola.

\section{Case Report}

A 36-years old male patient attended the emergency department of Luanda Medical Center, Angola, in February 2019, with fever $\left(39^{\circ} \mathrm{C}\right)$, pulse rate of 108 beats/minute, blood pressure of $128 / 69 \mathrm{mmHg}$, low back pain, discomfort during urination and chills since two weeks ago. The patient was empirically treated with Ciprofloxacin (500 mg orally every 12 hours) for urinary tract infection after asking for the necessary laboratory tests. The blood tests showed leukocytosis $\left(24.1 \times 10^{3}\right.$ cells/ $\mu \mathrm{L}$ with $85 \%$ neutrophils) and C-Reactive Protein (CRP) 45.5 $\mathrm{mg} / \mathrm{L}$. A routine urinalysis was performed using dipstick test and microscopic examination. The results showed more than 10 leucocytes per field and abundant bacteraemia in the sediment.

\subsection{Results}

The urine culture was performed on 5\% Sheep Blood and MacConkey agar plates. Round, smooth and dark violet pigmentation colonies (Figure 1) were grown on both media after $24 \mathrm{~h}$ of culture at $35^{\circ} \mathrm{C}$. They were oxidase positive, lactose non-fermenting Gram negative rods, and were identified as Chromobacterium violaceum using RemelRapID NF PLUS System.

\subsection{Antibiotic Susceptibility}

The antibiotic susceptibility was determined using the disc diffusion method on Mueller Hinton agar according to the Guidelines of the Clinical Laboratory Standards Institute (GCLSI) [6]. The C. violaceum was sensitive to Amoxicillin/Clavulanic acid, Ceftriaxon, Ciprofloxacin, Doxycycline, Trimethoprim/Sulfamethoxazole, Piperacilin/Tazobactam, Gentamicin, Amikacin, Aztreonam and Imipenem. The bacteria showed resistance to Cefuroxime (Figure 2).

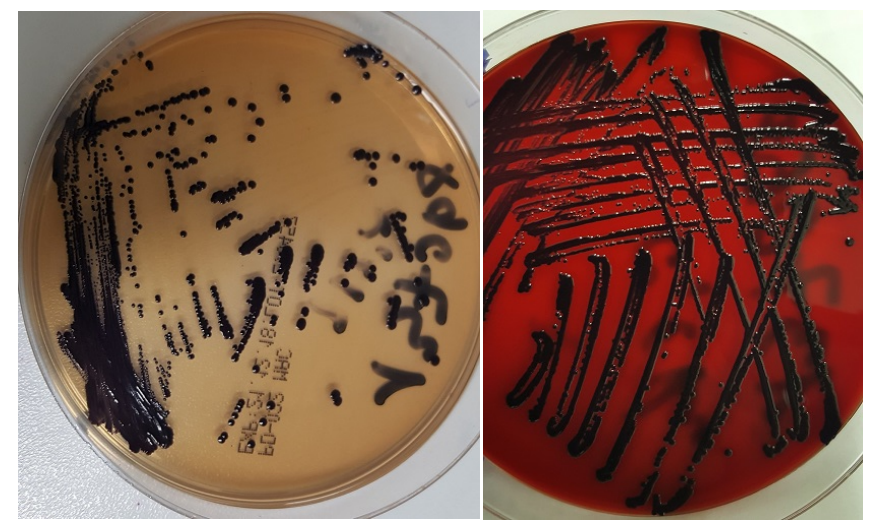

Figure 1. Growth of Chromobacterium violaceum on MacConkey (left) and 5\% Sheep blood agar (right). 


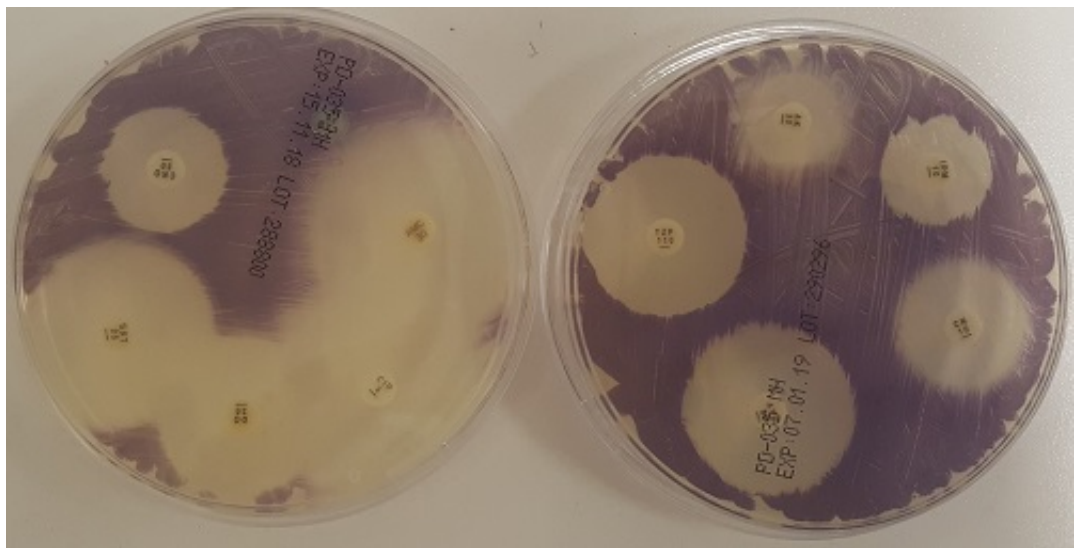

Figure 2. Antimicrobial susceptibility testing of Chromobacterium violaceum on Mueller Hinton agar.

Since the organism was found to be sensitive toward Ciprofloxacin, the initial antibiotic therapy was continued for 1 week. After this period of time, the clinical symptoms disappeared completely: white blood cells (WBC) in blood returned in normal level $\left(5.98 \times 10^{3}\right.$ cells $\left./ \mu \mathrm{L}\right)$ and the urine culture control was negative.

\section{Discussion}

The infection caused by $C$. violaceum is very rare, but with a high mortality rate [5] [7]. The review of the literature revealed only three cases of Chromobacterium infection in adults from Africa [5]. Until now there were no reports from Africa about urinary tract infection caused by $C$. violaceum. This is the first case of $C$. violaceum as UTI agent reported in Angola. The antimicrobial susceptibility pattern of $C$. violaceum is very limited due to the rarity of isolation from clinical specimens. $C$. violaceum is usually sensitive to Fluoroquinolones, Carbapenems, Aminoglycosides, Chloramphenicol, Trimethoprim-sulfamethoxazole, Tetracyclines, but resistant to Penicillin and Cephalosporins [7] [8]. After 1990, Ciprofloxacin and Carbapenems became the predominant antimicrobial agents [5] [9]. In this case the organism was susceptible to Ciprofloxacin, Trimethoprim-sulfamethoxazole, Doxycycline that corresponds to data obtained from others studies [3] [4] [10]. Also our study demonstrated the sensitivity of $C$. violaceum to Amoxicillin/Clavulanic acid while Swain [3] and Plant [4] reported resistance of $C$. violaceum to it. The bacteria enter into the body usually through a skin trauma or ingestion of contaminated water and seafood [1] [5] [11]. Our patient did not have any trauma and it is still not clear the pathway of this infection to urine tract system.

\section{Conclusion}

C. violaceum infection is a rare emerging infection that develops with a high mortal rate. If it is not treated properly, it may lead to death. The Ciprofloxacin has shown a good activity against $C$. violaceum infection. 


\section{Acknowledgements}

The authors express gratitude to Dr. Michael Averbukh, Dr. Aaron Cohen and all staff of the Luanda Medical Center for their help and support.

\section{Conflicts of Interest}

The authors declare that he has no competing interests.

\section{References}

[1] Koburger, J.A. and May, S.O. (1982) Isolation of Chromobacterium spp. from Foods, Soil, and Water. Applied and Environmental Microbiology, 44, 1463-1465.

[2] Jitmuang, A. (2008) Human Chromobacterium violaceum Infection in Southeast Asia: Case Reports and Literature Review. The Southeast Asian Journal of Tropical Medicine and Public Health, 39, 452-460.

[3] Swain, B., Otta, S., Sahu, K.K., et al. (2014) Urinary Tract Infection by Chromobacterium violaceum. Journal of Clinical and Diagnostic Research, 8, 1-2.

[4] Pant, N.D. and Sharma, M. (2015) Urinary Tract Infection Caused by Chromobacterium violaceum. International Journal of General Medicine, 8, 293-295. https://doi.org/10.2147/IJGM.S89886

[5] Yang, C.H. and Li, Y.H. (2011) Chromobacterium violaceum Infection: A Clinical Review of an Important but Neglected Infection. Journal of the Chinese Medical Association, 74, 435-441. https://doi.org/10.1016/j.jcma.2011.08.013

[6] Clinical Laboratory Standards Institute (2015) Performance Standards for Antimicrobial Susceptibility Testing. M100-S23.

[7] Anah, M.U., Udo, J.J., Ochigbo, S.O., et al. (2004) Neonatal Septicemia in Calabar, Nigeria. Tropical Doctor, 38, 126-128. https://doi.org/10.1258/td.2006.006037

[8] Pallab, R., Sharma, J., Rungmei, S.K., et al. (2004) Chromobacterium violaceum Septicaemia from North India. The Indian Journal of Medical Research, 120, 523-526.

[9] Aldridge, K.E., Valaninis, G.T. and Saners, C.V. (1988) Comparison of the in Vitro Activity of Ciprofloxacin and 24 Other Antimicrobial Agents against Clinical Strains of Chromobacterium violaceum. Diagnostic Microbiology and Infectious Disease, 10, 31-39. https://doi.org/10.1016/0732-8893(88)90124-1

[10] Kaniyarakkal, V., Orvankundil, S., Karunakaran, S., et al. (2016) Chromobacterium violaceum: Septicaemia and Urinary Tract Infection: Case Reports from a Tertiary Care Hospital in South India. Case Reports in Infectious Diseases, Article ID: 6795743.

[11] Byamukama, D., Farnleitner, A.H., Kansiime, F., et al. (2005) Contrasting Occurrence of Chromobacterium violaceum in Tropical Drinking Water Springs of Uganda. Journal of Water and Health, 3, 229-238. https://doi.org/10.2166/wh.2005.028 